\title{
Switching of Ticagrelor to Clopidogrel at 3 Months in Patients Treated for Acute Coronary Syndrome; Single Centre Experience
}

\section{Tahir Hamid', Mahvash Zaman', Samman Rose ${ }^{1}$ and Nadim Malik ${ }^{1,2 *}$}

${ }^{1}$ Department of Cardiology, Stepping Hill Hospital, Stockport NHS Foundation Trust, UK ${ }^{2}$ University of Manchester, UK

\begin{abstract}
Objective: ESC guidelines recommend Dual Anti-Platelet Therapy (DAPT) with aspirin and ticagrelor for 12 months following Non-ST Elevation Myocardial Infarction (NSTEMI). We aimed to assess the safety of switching DAPT with aspirin and ticagrelor to aspirin and clopidogrel after 3 months in patients following NSTEMI, with the maximum duration of DAPT of 12 months.

Material and methods: Patients admitted with NSTEMI between 2011-2012 were identified using the ICD-10 and OPCS-4 coding systems. Retrospective analysis was then performed using electronic records for additional information.

Results: 98 patients were treated with aspirin and ticagrelor following admission with Ml. $64 \%(63 / 98)$ were male, $55.1 \%(54 / 98)$ were hypertensive, $66.3 \%$ (65/98) with hyperlipidemia, $20.4 \%(20 / 98)$, had diabetes and $33.7 \%$ (33/98) had previous known ischemic heart disease, 40.8\% (40/98) were ex-smokers, 35.7\% (35/98) had BMI > 30. $74.5 \%$ (73/98) underwent percutaneous coronary intervention with stenting of the target lesions, $20.4 \%(20 / 98)$ treated medically while $4.1 \%(4 / 98)$ referred for coronary bypass surgery. $8.2 \%(8 / 98)$ patients were re-admitted within 90 days of NSTEMI before the switchover of DAPT ( 3 for angina, 2 for non-cardiac chest pains and 3 for non-cardiac conditions), and none after that period. In $51 \%$ (50/98) patients DAPT was switched to clopidogrel at 3 months with $49 \%(48 / 98)$ staying on aspirin and ticagrelor. There were three non-cardiac deaths in the follow-up period.

Conclusion: This study shows the potential for the safe switchover of DAPT to clopidogrel following 3 months therapy with ticagrelor for NSTEMI, whilst enhancing cost-savings.
\end{abstract}

Keywords: Ticagrelor; Clopidogrel; Acute coronary syndrome

\section{Introduction}

Treatment with dual antiplatelet therapy including aspirin and a P2Y12 inhibitor is gold standard therapy in the management of patients with Acute Coronary Syndromes (ACS) [1,2]. Ticagrelor, a reversible oral $\mathrm{P} 2 \mathrm{Y} 12$ receptor antagonist provides faster, greater and consistent platelet inhibition than Prasugrel and clopidogrel [3-5]. In the PLATO trial (The Platelet Inhibition and Patient Outcomes) [6], ticagrelor demonstrated superiority in preventing death from cardiovascular causes, non-fatal MI and stroke and without an increase in rate of overall major bleeding. Prasugrel is an irreversible P2Y12 inhibitor. Prasugrel has shown reduced ischemic events in patients with ACS planned for Percutaneous Coronary Interventions (PCI) in the TRITON Trial [7], however it showed no benefit over clopidogrel in Non-ST-Elevation Acute Coronary Syndrome (NSTE-ACS) patients who are for management without revascularization (TRILOGY-ACS trial) [8]. 12 months of Dual-Antiplatelet Therapy (DAPT) with a P2Y12 inhibitor and aspirin is accepted to offer a more clinicallyeffective option than treatment with clopidogrel and aspirin, however there has been little research into the switching of anti-platelet agents, especially from ticagrelor to clopidogrel, at intervals earlier than 12 months.

Occasionally, in clinical scenarios such as following the onset of 'unpleasant' side effects associated with the newer P2Y12 inhibitors, the recommendation is to switch DAPT. Such 'switching' of DAPT is often to the older clopidogrel-aspirin combination. Admittedly, such 'switchover' of DAPT is based on case-by-case recommendation of the supervising clinician and is rather uncommon. Nevertheless, there are no reported adverse outcomes from such 'downgrading' of DAPT after an initial period of more aggressive DAPT.
Based on these observations, "the downgrading of DAPT" to the older 'clopidogrel-aspirin' combination, after initiation of the newer DAPT regimes warrants further investigation, especially as it would offer possible clinical and financial advantages. We hypothesized that in patients admitted with a clinical presentation of Ischaemic Heart Disease (IHD), a 'switchover' of DAPT from 'ticagrelor-aspirin' to 'clopidogrel-aspirin' regime after a 3-month period would not be associated with any adverse clinical outcomes (MACE or ischaemic complications).

\section{Methods}

\section{Study population}

The data source is an observational retrospective analysis from a prospective data registry of 98 patients who were treated with ticagrelor following admission with Acute Coronary Syndrome (ACS) between 2011-2012 in a single hospital in UK. Patients were identified using the ICD-10 and OPCS-4 coding system. All patients were treated for ACS according to NICE guidelines, and as per agreed local policy at that

*Corresponding author: Nadim Malik, Consultant Cardiologist, Department of Cardiology, Stepping Hill Hospital, Stockport NHS Foundation Trust, SK2 7JE, UK, Tel: 0161483 1010; E-mail: Nadim.malik@manchester.ac.uk

Received July 02, 2016; Accepted August 23, 2016; Published August 29, 2016

Citation: Hamid T, Zaman M, Rose S, Malik N (2016) Switching of Ticagrelor to Clopidogrel at 3 Months in Patients Treated for Acute Coronary Syndrome; Single Centre Experience. Cardiovasc Pharm Open Access 5: 194. doi: 10.4172/23296607.1000194

Copyright: @ 2016 Hamid T, et al. This is an open-access article distributed under the terms of the Creative Commons Attribution License, which permits unrestricted use, distribution, and reproduction in any medium, provided the original author and source are credited. 
time whereby approval for ticagrelor therapy was limited to 3 months only due to high cost. Additional information was collected through follow-up clinic letters, patient electronic records and procedural databases. Recorded data fields included patient demographics, comorbidities, treatment strategy, duration and type of DAPT, hospital re-admissions and mortality.

Patients admitted with ACS were treated with a loading dose of aspirin $300 \mathrm{mg}$, followed by $75 \mathrm{mg}$ once daily. The second antiplatelet agent was ticagrelor with a loading dose of $180 \mathrm{mg}$ followed by $90 \mathrm{mg}$ twice daily. In addition to optimal medical therapy, patient were treated with Percutaneous Coronary Interventions (PCI) with Bare Metal or Drug-Eluting Stents based on standard practice of the operator, surgical revascularization with Coronary Artery By-Pass Surgery (CABG) or medical therapy only.

\section{Clinical definitions}

All deaths were considered as cardiac unless a specific alternate cause of death was demonstrated.

Post procedural MI was defined as the presence of at least 2 of the following: Cardiac chest pains, rise in cardiac enzymes (creatine kinase or troponin) and new ECG changes.

MACE was defined as; all-cause mortality, cardiac mortality, myocardial infarction (MI), stroke and Target Vessel Revascularization (TVR).

The Target Lesion Revascularization (TLR) was defined as a repeated intervention within the stent or in the immediate distal or proximal segments adjacent to the stent.

TVR was defined as any repeat PCI or CABG of the target vessel.

The successful PCI for the target coronary lesion was defined as restoration of TIMI flow grade 3 without major complications after stent deployment.

Follow-up time was defined as the interval from the index procedure to the last contact.

\section{Follow-up}

Patients were followed up routinely at 3-6 months and/or until discharged (discretion of clinician) to the community based on the symptoms. At follow-up patients had a history review and clinical examination. Re-admissions with cardiac events were checked using hospital electronic records, catheter-lab database, and all other clinical letters (admissions \& outpatients).

Statistical analyses were performed using SPSS $20^{\circ}$ software (SPSS Inc., Chicago, IL, USA). A p-value of $<0.05$ was considered statistically significant.

\section{Results}

98 patients with a mean age of 67.7 years were treated with aspirin and ticagrelor following admission with MI as per guidelines. Patient's demographics are shown in Table 1.

Treatment strategy varied amongst patients; 73 (74.5\%) underwent PCI with stenting of the target lesions. 20 (20.4\%) were treated medically while 4 (4.1\%) were referred for CABG. In 50 patients $(51 \%)$ DAPT was switched to aspirin and clopidogrel at 3 months (Group 2), and the remaining 48 patients (49\%) stayed on aspirin and ticagrelor (Group 1) for a total of 12 months. The medications were changed by the local family physicians as per local policy at the time.

In total, 9 patients $(8.2 \%)$ were re-admitted within 90 days of NSTE-ACS, before the switchover of DAPT (3 for angina and 6 with non-coronary related conditions) and none after that period. $4 / 9$ (44\%) patients had been treated with PCI, 4 (44\%) treated medically and 1 (11\%) treated with CABG. At full follow up of the study population, there were 3 deaths (3\%) (All of these patients were $>80$ years old, treated medically and had non-cardiac deaths). There was no other documented MACE (MI, TVR, stroke, major bleed) recorded in either group (ticagrelor vs clopidogrel).

\section{Discussion}

Currently both NICE and ESC guidelines recommend aspirin and a P2Y12 inhibitor (clopidogrel/ticagrelor/Prasugrel) as DAPT for 12 months post ACS. The newer antiplatelets have significant cost implications, as they are considerably more expensive. We report absence of any significant adverse outcomes in an observational allcomer study of patients admitted with a clinical presentation of IHD following the 'switchover' of DAPT after a 3 month period of aggressive DAPT with the newer P2Y12 inhibitor (ticagrelor). In particular, the downgrading of DAPT to clopidogrel-aspirin did not lead to any significant ischaemic complications.

It is plausible that there are some significant differences between the active/unstable forms of IHD and the stable form of IHD. For example, it is acknowledged that during the period of acute presentation of IHD, there is a high level of residual platelet and thrombin activation, leading to a pro-thrombotic/coagulant coronary state, and that this would be significantly less once the disease has reached a more stable status following achievement of improved coronary flow from the combination of optimal medical and mechanical revascularization therapies [9-12]. The fact that patients in the present study, across all three treatment groups; medical therapy only, PCI with stents and CABG, benefitted from the 'switchover' would support achievement of a stable IHD status as being the main influence for this regardless of the supporting revascularization strategy. Admittedly, therefore, whilst the acute and unstable nature of the IHD at clinical presentation would benefit from the more aggressive and greater platelet inhibition, in the form of the newer P2Y12 antagonists, the stabilized IHD disease (after 3 months) would not necessarily require the same 'more aggressive' therapy. The clopidogrel-aspirin' combination may therefore be sufficient, after 3 months, in maintaining adequate level of platelet inhibition. Our study data supports such a possibility without any concerns regarding patient safety i.e., adverse MACE outcomes from such a 'switchover'.

Second, it is not uncommon to rationalize the need for more aggressive DAPT regime in patients following PCI, especially in view of an enhanced risk of 'stent thrombosis'. It is now widely acknowledged that in cases that re-present with 'stent thrombosis' following PCI whilst on DAPT, it is often not just the underlying disease and the procoagulant state of blood flow, but also PCI-related factors that influence such re-admissions. Therefore, whilst the choice of more aggressive DAPT with newer P2Y12 antagonists would be an important adjuvant for such scenarios, the underlying issues of untreated residual disease, small edge dissections, possible inadvertent inadequate stent expansion and perhaps the use of extensive stent metal (long stents, stent overlap, bifurcation stenting etc.) also need addressing (more than the choice of switching to aggressive DAPT regime). Nevertheless, once the early period has lapsed, it might still be possible to switchover from a 'more aggressive' to the 'less aggressive' DAPT regime without jeopardizing 


\begin{tabular}{|c|c|c|c|}
\hline & Group A (12 months ticagrelor) & $\begin{array}{l}\text { Group B } \quad \text { (Switching to } \\
\text { clopidogrel after } 3 \text { months) }\end{array}$ & \\
\hline Patient No. & 50 & 48 & - \\
\hline Male & $22(44.0 \%)$ & $41(85.4 \%)$ & - \\
\hline Hypertension & $22(44.0 \%)$ & $32(66.6 \%)$ & - \\
\hline Hyperlipidemia & $32(64.0 \%)$ & $33(68.75 \%)$ & - \\
\hline Diabetes & $11(22.0 \%)$ & $9(18.75 \%)$ & - \\
\hline Previous Known IHD & $21(42.0 \%)$ & $12(25.0 \%)$ & - \\
\hline Ex-smokers & $21(42.0 \%)$ & $19(39.5 \%)$ & - \\
\hline $\mathrm{BMI}>25$ & $25(50 \%)$ & $31(64.5 \%)$ & - \\
\hline Mortality & $1(2.1 \%)$ & $2(4.0 \%)$ & $(p=1.0)$ \\
\hline \multicolumn{4}{|c|}{ IHD: Ischaemic Heart Disease; BMI: Body Mass Index } \\
\hline
\end{tabular}

Table 1: Patient's demographics.

clinical outcome. Once again, our data implies that such a 'switchover' of DAPT therapy to maintain less intense platelet inhibition might not necessarily be unsafe after 3 months when the localized coronary injury site is well on its way to healing.

Third, in some cases, there is a greater need for tailoring the antiplatelet regime to the individual patient. For example, the very elderly ( $>80$ years) and frail patients are often acknowledged to be at higher risk of bleeding. For this reason alone, DAPT with Prasugrel in the elderly ( $>75$ years) and the frail ( $<60 \mathrm{~kg}$ weight) is not recommended. DAPT with ticagrelor, whilst not contraindicated in such high-risk patients, is often still used with caution. Likewise, the use of the newer P2Y12 inhibitors in patients on formal anti-coagulant regimes (Warfarin/New Oral Anticoagulants (NOACs) used in conditions such as systemic thrombosis, chronic atrial fibrillation, prosthetic valve disease etc.) remains unclear. Longer periods of more aggressive DAPT (ticagreloraspirin or Prasugral-aspirin) are often not considered in such cases. In addition, even after newer DAPT regimes have been initiated in some such cases, there is lack of consensus on the duration of such DAPT as well as whether it should be with or without formal anticoagulant use (i.e., triple therapy). Finally, the question of when to initiate the 'switchover' to less intense platelet inhibition in such cases on formal anti-coagulant regimes is yet to be answered. Often in cases where there is a need to continue or even resume formal anticoagulation, the choice is in favor of antiplatelet monotherapy, and rarely DAPT with newer P2Y12 inhibitors. More often, clopidogrel-aspirin is used, but again for a short duration of a few months only. The data from the present study, whilst not specifically looking at such high-risk patients, does however suggest the safety of such 'switchover' after a period of 3 months.

The optimal dosing and timing of switching after the last dose of ticagrelor to clopidogrel is unclear. It has been found that a loading dose of clopidogrel reduces the risk of High On-Treatment Platelet Reactivity (HTPR) with no incrase in bleeding risk [13]. A waiting period of $24 \mathrm{~h}$ after the last dose of ticagrelor before loading with 600 $\mathrm{mg}$ of clopidogrel has been suggested in literature to allow sufficient time for ticagrelor and its metabolites to be eliminated as well as for new platelets to be exposed to the active metabolite of clopidogrel. Data in this area is very limited [14].

One year treatment with ticagrelor has been associated with an approximate 0.18 and 0.16 gain in Life-Years (LY) and QualityAdjusted Life Years (QALY) respectively $[15,16]$. However, ticagrelor comes at a cost; with a 28-pack of ticagrelor costing $£ 54.60$ compared to a 30-pack of clopidogrel at $£ 3.40$. This additive cost to cash-strapped public services such as the National Health Service (NHS) in the UK is of particular importance. By switching over from ticagrelor to clopidogrel at 3 months, this results in a $£ 1042.00$ saving per patient per annum.
This study highlights the potential for safely switching from ticagrelor to clopidogrel at an interval of 3 months post ACS. This would be a more economically viable option in the current financially stretched institutes, which may in turn lead to the delivery of better healthcare as it would reduce the funding issues associated with ticagrelor, and enable more patients to receive it as treatment post ACS.

\section{Limitations of Study}

This is a non-randomized retrospective observational registry of a single center experience. The statistical data derived from such a small study sample population would not be without its limitations. However, it would be sufficient to rationalize a recommendation for a larger trial on the issue of 'switchover' of DAPT from the newer more expensive and aggressive therapies to the cheaper but older DAPT regime. A longer-term follow up (MACE outcomes) beyond 1 year would also be necessary to ensure long-term safety of a 'switchover' regime.

\section{Conclusion}

This study demonstrates the potential for safe switchover of DAPT to aspirin and clopidogrel following initial 3-month therapy with aspirin and ticagrelor independent of the revascularization treatment strategy. This would enhance cost-savings with no compromise in clinical care.

\section{References}

1. Hamm CW, Bassand JP, Agewall S, Bax J, Boersma E, et al. (2011) ESC Guidelines for the management of acute coronary syndromes in patients presenting without persistent ST-segment elevation: The Task Force for the management of acute coronary syndromes (ACS) in patients presenting without persistent ST-segment elevation of the European Society of Cardiology (ESC). Eur Heart J 32: 2999-3054.

2. Wright RS, Anderson JL, Adams CD, Antman EM, Bridges CR, et al. (2011) 2011 ACCF/AHA focused update incorporated into the ACC/AHA 2007 Guidelines for the Management of Patients with Unstable Angina/Non-STElevation Myocardial Infarction. J Am Coll Cardiol 57: e215-e367.

3. Storey RF, Husted S, Harrington R, Heptinstall S, Wilcox RG, et al. (2007) Inhibition of platelet aggregation by AZD6140, a reversible oral P2Y12 receptor antagonist, compared with clopidogrel in patients with acute coronary syndromes. J Am Coll Cardiol 50: 1852-1856.

4. Gurbel PA, Bliden KP, Butler K, Tantry US, Gesheff T, et al. (2009) Randomized double-blind assessment of the ONSET and OFFSET of the antiplatelet effects of ticagrelor vs. clopidogrel in patients with stable coronary artery disease: the ONSET/OFFSET study. Circulation 120: 2577-2585.

5. Gurbel PA, Bliden KP, Butler K, Antonino MJ, Wei C, et al. (2010) Response to ticagrelor in clopidogrel nonresponders and responders and effect of switching therapies: the RESPOND study. Circulation 121: 1188-1199.

6. Wallentin L, Becker RC, Budaj A, Cannon CP, Emanuelsson H, et al. (2009) Ticagrelor versus clopidogrel in patients with acute coronary syndromes. $\mathrm{N}$ Engl J Med 361: 1045-1057. 
Citation: Hamid T, Zaman M, Rose S, Malik N (2016) Switching of Ticagrelor to Clopidogrel at 3 Months in Patients Treated for Acute Coronary Syndrome; Single Centre Experience. Cardiovasc Pharm Open Access 5: 194. doi: 10.4172/2329-6607.1000194

7. Wiviott SD, Braunwald E, McCabe CH, Montalescot G, Ruzyllo W, et al. (2007) Prasugrel versus clopidogrel in patients with acute coronary syndromes. N Engl J Med 357: 2001-2015.

8. Roe MT, Armstrong PW, Fox KAA, White HD, Prabhakaran D, et al. (2012) Prasugrel versus clopidogrel for acute coronary syndromes without revascularization. N Engl J Med 367: 1297-1309.

9. Rade JJ (2012) Platelet function testing in patients with coronary artery disease. Is the who and the when any clearer than the what and the what then? Circulation 3073-3075.

10. Gluckman TJ, McLean RC, Schulman SP, Kickler TS, Shapiro EP, et al. (2011) Effects of aspirin responsiveness and platelet reactivity on early vein graft thrombosis after coronary artery bypass graft surgery. J Am Coll Cardiol 57 : 1069-1077.

11. Gurbel PA, Bliden KP, Hiatt BL, O'Connor CM (2003) Clopidogrel for coronary stenting: response variability, drug resistance, and the effect of pretreatment platelet reactivity. Circulation 107: 2908-2913.
12. Price MJ, Berger PB, Teirstein PS, Tanguay JF, Angiolillo DJ, et al. (2011) Standard- vs high-dose clopidogrel based on platelet function testing after percutaneous coronary intervention: the GRAVITAS randomized trial. JAMA 305: $1097-1105$

13. Pourdjabber A, Hibbert H, Simard T, Ramirez FD, Lugomirski P, et al. (2015) Optimizing crossover from ticagrelor to clopidogrel in patients with acute coronary syndrome: the capital opti-cross trial. J Am Coll Cardiol 65: A108.

14. Rollini F, Franchi F, Angiolillo D (2016) Switching P2Y12-receptor inhibitors in patients with coronary artery disease. Nat Rev Cardiol 13: 11-27.

15. Thiedel U, Asseburg C, Giannitsis E, Katus H (2013) Cost-effectiveness of ticagrelor versus clopidogrel for the prevention of atherothrombotic events in adult patients with acute coronary syndrome in Germany. Clin Res Cardiol 102 : 446-458.

16. Crespin DJ, Federspiel JJ, Biddle AK, Jonas DE, Rossi JS (2011) Ticagrelor versus genotype-driven antiplatelet therapy for secondary prevention after acute coronary syndrome: a cost-effectiveness analysis. Value Health 14 483-491.
Citation: Hamid T, Zaman M, Rose S, Malik N (2016) Switching of Ticagrelo to Clopidogrel at 3 Months in Patients Treated for Acute Coronary Syndrome; Single Centre Experience. Cardiovasc Pharm Open Access 5: 194. doi: $10.4172 / 2329-6607.1000194$ 